\title{
Sampling-based learning control of inhomogeneous quantum ensembles
}

\author{
Chunlin Chen, ${ }^{1,2}$ Daoyi Dong, ${ }^{3,}{ }^{*}$ Ruixing Long, ${ }^{2}$ Ian R. Petersen, ${ }^{3}$ and Herschel A. Rabitz ${ }^{2}$ \\ ${ }^{1}$ Department of Control and System Engineering, Nanjing University, Nanjing 210093, China \\ ${ }^{2}$ Department of Chemistry, Princeton University, Princeton, New Jersey 08544, USA \\ ${ }^{3}$ School of Engineering and Information Technology, University of New South Wales at the Australian Defence Force Academy, \\ Canberra, Australian Capital Territory 2600, Australia \\ (Received 30 August 2013; published 5 February 2014)
}

\begin{abstract}
Compensation for parameter dispersion is a significant challenge for control of inhomogeneous quantum ensembles. In this paper, we present the systematic methodology of sampling-based learning control (SLC) for simultaneously steering the members of inhomogeneous quantum ensembles to the same desired state. The SLC method is employed for optimal control of the state-to-state transition probability for inhomogeneous quantum ensembles of spins as well as $\Lambda$-type atomic systems. The procedure involves the steps of (i) training and (ii) testing. In the training step, a generalized system is constructed by sampling members according to the distribution of inhomogeneous parameters drawn from the ensemble. A gradient flow based learning and optimization algorithm is adopted to find an optimal control for the generalized system. In the process of testing, a number of additional ensemble members are randomly selected to evaluate the control performance. Numerical results are presented, showing the effectiveness of the SLC method.
\end{abstract}

DOI: 10.1103/PhysRevA.89.023402

PACS number(s): 03.67.-a, 02.30.Yy, 03.67.Pp

\section{INTRODUCTION}

Control of quantum phenomena lies at the heart of emerging quantum technology [1-5]. Quantum control theory has many components including controllability assessment, optimal control, feedback control, etc. Most existing results focus on control design of single quantum systems [1-19]. Another important issue is control design for quantum ensembles. A quantum ensemble consists of a large number (up to $\sim 10^{23}$ ) of single quantum systems (e.g., spin systems), and every quantum system is referred to as a member of the ensemble. This paper considers an ensemble in the sense of the individual systems slightly varying over a distribution of characteristics, rather than in the content of a mixed state. Quantum ensembles have wide applications in emerging quantum technology including quantum computation [20], long-distance quantum communication [21], quantum memory [22], and magneticresonance imaging [23]. Several results on quantum ensemble control have been presented including unitary control in homogeneous quantum ensembles for maximizing signal intensity in coherent spectroscopy [24] and feedback stabilization of quantum ensembles [25].

In practical applications, the members of a quantum ensemble could have variations in the parameters that characterize the system dynamics $[26,27]$. For example, the spins of an ensemble in nuclear magnetic-resonance (NMR) experiments may encounter large dispersion in the strength of the applied radio frequency field (rf inhomogeneity) as well as the members exhibiting variations in their natural frequencies (Larmor dispersion) $[28,29]$. In this paper, these situations are referred to as inhomogeneous quantum ensembles. It is generally impractical to employ different control inputs for individual members of a quantum ensemble in the laboratory. Hence, it is important to develop the means for designing control fields that can simultaneously steer the ensemble of systems

*daoyidong@gmail.com from an initial state to a desired target state when variations exist in the system parameters. Such controls are also called compensating pulse sequences in NMR spectroscopy [26,30]. Other applications include control of a randomly oriented ensemble of molecules in physical chemistry [31], the design of slice selective excitation and inversion pulses in magneticresonance imaging, and the correction of systematic errors in quantum information processing [27]. Theoretical results show that under commonly arising conditions there exist optimal laser fields to control all molecules in an inhomogeneous ensemble, regardless of their orientation or spatial location $[32,33]$. Recent studies considered the controllability and optimal control of inhomogeneous spin ensembles [23,27,3437]. An additional investigation examined the stabilization of an inhomogeneous ensemble of noninteracting spin systems using Lyapunov control methodology [38]. However, a systematic method is lacking for designing effective control fields that can simultaneously steer the members of an inhomogeneous quantum ensemble to the same target state.

This paper presents a systematic methodology for control design of inhomogeneous quantum ensembles for the state-tostate transition probability, illustrated for spins and three-level $\Lambda$-type systems. The proposed method involves the steps of (i) training and (ii) testing, which we call sampling-based learning control (SLC). In the training step, we sample several members according to the distribution of inhomogeneous parameters from the ensemble and construct a generalized system using these collective samples. Then, we employ a gradient flow based learning and optimization algorithm [39] to find the control providing good performance for the generalized system. In the process of testing the deduced controls, we randomly select a number of sampling members to evaluate the control performance. Numerical simulations show that the SLC method has potential for practical control design of various inhomogeneous quantum ensembles. These findings support the previous theoretical analysis, suggesting that control of inhomogeneous ensembles should generally be feasible [31-34]. 
The paper is organized as follows. Section II formulates the control problem for inhomogeneous quantum ensembles and presents the details of SLC. The SLC method is illustrated for two-level and three-level inhomogeneous quantum ensembles, respectively, in Secs. III and IV. Conclusions are presented in Sec. V.

\section{METHODOLOGY}

\section{A. Model and problem formulation}

Consider a finite-dimensional closed quantum system with the evolution of its state $|\psi(t)\rangle$ described by the Schrödinger equation (setting $\hbar=1$ ):

$$
\begin{aligned}
& \frac{d}{d t}|\psi(t)\rangle=-i H(t)|\psi(t)\rangle, \\
& t \in[0, T], \quad|\psi(0)\rangle=\left|\psi_{0}\right\rangle .
\end{aligned}
$$

The solution of Eq. (1) is given by $|\psi(t)\rangle=U(t)\left|\psi_{0}\right\rangle$, where the propagator $U(t)$ satisfies

$$
\begin{aligned}
& \frac{d}{d t} U(t)=-i H(t) U(t), \\
& t \in[0, T], \quad U(0)=\mathrm{Id} .
\end{aligned}
$$

In this paper, we consider an inhomogeneous ensemble in which the Hamiltonian of each member has the following form:

$$
H_{\omega, \theta}(t)=g(\omega) H_{0}+b(\theta) \sum_{m=1}^{M} u_{m}(t) H_{m},
$$

where $H_{0}$ is the free Hamiltonian and $\sum_{m=1}^{M} u_{m}(t) H_{m}$ corresponds to the time-dependent control Hamiltonian that represents the interaction of the system with the external fields $u_{m}(t)$ (real-valued and square-integrable functions) through the Hermitian operators $H_{m}$. The functions $g(\omega)$ and $b(\theta)$ characterize the inhomogeneous distribution in the free Hamiltonian and control Hamiltonian, respectively (see Fig. 1). In this paper, we assume that $g(\omega)=\omega$ and $b(\theta)=\theta$, and the parameters $\omega$ and $\theta$ are time independent and uni-

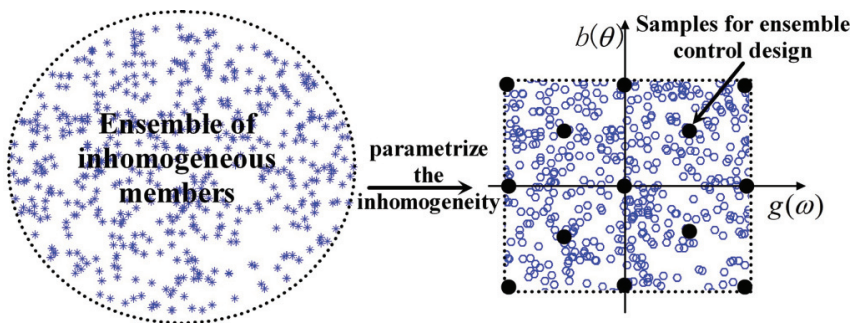

FIG. 1. (Color online) A schematic for modeling an inhomogeneous ensemble parametrized with $g(\omega)$ and $b(\theta)$, and the selection of samples to construct a generalized system. An ensemble of inhomogeneous members on the left is mapped into a space determined by $g(\omega)$ and $b(\theta)$ on the right, where $g(\omega)$ and $b(\theta)$ characterize the distribution of inhomogeneity in the free Hamiltonian and control Hamiltonian, respectively. Samples for ensemble control design are drawn from the distribution of inhomogeneous parameters. These samples are used to construct a generalized system for learning a control field with the performance as an average on the samples. formly distributed over $[1-\Omega, 1+\Omega]$ and $[1-\Theta, 1+\Theta]$, respectively. The constants $\Omega \in[0,1]$ and $\Theta \in[0,1]$ represent the bounds of the parameter dispersion. The objective is to design the controls $\left\{u_{m}(t), m=1,2, \ldots, M\right\}$ to simultaneously drive the members (with different $\omega$ and $\theta$ ) of the quantum ensemble from an initial state $\left|\psi_{0}\right\rangle$ to the same target state $\left|\psi_{\text {target }}\right\rangle$ with high fidelity. The control outcome is described by a performance function $J(u)$ for each control strategy $u=\left\{u_{m}(t), m=1,2, \ldots, M\right\}$. The control problem can then be formulated as a maximization problem as follows:

$$
\begin{aligned}
\max _{u} J(u) & :=\max _{u} \mathbb{E}\left[J_{\omega, \theta}(u)\right], \\
\text { such that } \frac{d}{d t}\left|\psi_{\omega, \theta}(t)\right\rangle & =-i H_{\omega, \theta}(t)|\psi(t)\rangle, t \in[0, T], \\
\left|\psi_{\omega, \theta}(0)\right\rangle & =\left|\psi_{0}\right\rangle, \\
H_{\omega, \theta}(t) & =\omega H_{0}+\theta \sum_{m=1}^{M} u_{m}(t) H_{m}, \\
\omega & \in[1-\Omega, 1+\Omega], \\
\theta & \in[1-\Theta, 1+\Theta],
\end{aligned}
$$

where $J_{\omega, \theta}(u)$ is the fidelity measure of each member of the ensemble and $\mathbb{E}\left[J_{\omega, \theta}(u)\right]$ denotes the average value of $J_{\omega, \theta}$ over the ensemble. The fidelity between the final state $\left|\psi_{\omega, \theta}(T)\right\rangle$ and the target state $\left|\psi_{\text {target }}\right\rangle$ is defined as follows [40]:

$$
F\left(\left|\psi_{\omega, \theta}(T)\right\rangle,\left|\psi_{\text {target }}\right\rangle\right)=\left|\left\langle\psi_{\omega, \theta}(T) \mid \psi_{\text {target }}\right\rangle\right| .
$$

The fidelity $F$ is used to evaluate the performance of a designed control in the testing step. However, for convenient calculation of a gradient flow in the training step, we take the performance function $J(u)=F^{2}$; i.e.,

$$
J_{\omega, \theta}(u):=\left|\left\langle\psi_{\omega, \theta}(T) \mid \psi_{\text {target }}\right\rangle\right|^{2} .
$$

Note that $J_{\omega, \theta}$ depends implicitly on the control $u$ through the Schrödinger equation.

\section{B. Sampling-based learning control of inhomogeneous quantum ensembles}

Gradient-based methods [5,39,4142] have been successfully applied to search for optimal solutions to a variety of quantum control problems, including in theoretical and laboratory applications. In this paper, a gradient-based learning method is employed to optimize the controls for inhomogeneous quantum ensembles. We present a systematic methodology for ensemble control design utilizing selected samples (as shown in Fig. 1) from the ensemble. These samples are drawn from the distribution of inhomogeneous parameters to design the control. Then the resultant control is applied to additional ensemble members to test the control performance. As such, the SLC method includes the steps of (i) training and (ii) testing.

\section{Training}

In the training step, we select $N$ sampled members from the quantum ensemble according to the distribution (e.g., uniform distribution) of the inhomogeneous parameters and 
then construct a generalized system as follows:

$$
\frac{d}{d t}\left(\begin{array}{c}
\left|\psi_{\omega_{1}, \theta_{1}}(t)\right\rangle \\
\left|\psi_{\omega_{2}, \theta_{2}}(t)\right\rangle \\
\vdots \\
\left|\psi_{\omega_{N}, \theta_{N}}(t)\right\rangle
\end{array}\right)=-i\left(\begin{array}{c}
H_{\omega_{1}, \theta_{1}}(t)\left|\psi_{\omega_{1}, \theta_{1}}(t)\right\rangle \\
H_{\omega_{2}, \theta_{2}}(t)\left|\psi_{\omega_{2}, \theta_{2}}(t)\right\rangle \\
\vdots \\
H_{\omega_{N}, \theta_{N}}(t)\left|\psi_{\omega_{N}, \theta_{N}}(t)\right\rangle
\end{array}\right)
$$

where $\quad H_{\omega_{n}, \theta_{n}}=\omega_{n} H_{0}+\theta_{n} \sum_{m} u_{m}(t) H_{m} \quad$ with $\quad n=$ $1,2, \ldots, N$. The performance function for the generalized system is defined by

$$
J_{N}(u):=\frac{1}{N} \sum_{n=1}^{N} J_{\omega_{n}, \theta_{n}}(u)=\frac{1}{N} \sum_{n=1}^{N}\left|\left\langle\psi_{\omega_{n}, \theta_{n}}(T) \mid \psi_{\text {target }}\right\rangle\right|^{2} .
$$

The goal of the training step is to find a control $u^{*}$ that maximizes the performance function defined in Eq. (7). The performance function is $J_{N}\left(u^{0}\right)$ with an initial control $u^{0}=$ $\left\{u_{m}^{0}(t)\right\}$. We apply the gradient flow method [5,29,39-42] to seek an optimal control $u^{*}=\left\{u_{m}^{*}(t)\right\}$. The detailed gradient flow algorithm is provided in the Appendix (Algorithm 1). The time interval $[0, T]$ is divided equally into $Q$ time slices $\triangle t$, and we assume that the controls are constant within each time slice. The time index is $t_{q}=q T / Q$, where $Q=T / \triangle t$ and $q=1,2, \ldots, Q$.

The motivation behind SLC is to design the control using a minimal number of sample members. Therefore, it is necessary to choose a representative set of samples. For example, when the distributions of both $\omega$ and $\theta$ are uniform, we may choose equally spaced samples in the $\omega-\theta$ space. In this case, the intervals of $[1-\Omega, 1+\Omega]$ and $[1-\Theta, 1+\Theta]$ are divided into $N_{\Omega}+1$ and $N_{\Theta}+1$ subintervals, respectively, where $N_{\Omega}$ and $N_{\Theta}$ are conveniently chosen positive odd integers. Then the total number of samples is $N=N_{\Omega} N_{\Theta}$, where $\omega_{n}$ and $\theta_{n}$ are chosen from all combinations of $\left(\omega_{n_{\Omega}}, \theta_{n_{\Theta}}\right)$ as follows:

$$
\begin{aligned}
& \omega_{n} \in\left\{\omega_{n_{\Omega}}=1-\Omega+\frac{\left(2 n_{\Omega}-1\right) \Omega}{N_{\Omega}}, n_{\Omega}=1,2, \ldots, N_{\Omega}\right\}, \\
& \theta_{n} \in\left\{\theta_{n_{\Theta}}=1-\Theta+\frac{\left(2 n_{\Theta}-1\right) \Theta}{N_{\Theta}}, n_{\Theta}=1,2, \ldots, N_{\Theta}\right\} .
\end{aligned}
$$

\section{Testing}

For testing, we apply the optimal control $u^{*}$ obtained in the training step to additional samples randomly selected from the inhomogeneous quantum ensemble and evaluate the control performance of each sample in terms of the fidelity $F\left(|\psi(T)\rangle,\left|\psi_{\text {target }}\right\rangle\right)$ between the final state achieved by each sample $|\psi(T)\rangle$ and the target state $\left|\psi_{\text {target }}\right\rangle$. If both the average value and the minimum value of the fidelity $F\left(|\psi(T)\rangle,\left|\psi_{\text {target }}\right\rangle\right)$ for all the tested samples are satisfactory, we accept the designed control law and end the control design process. Otherwise, we return to the training step and generate another optimized control strategy (e.g., restarting the training step with a new initial control strategy or a new set of samples guided by the performance of the tested members).

\section{CONTROL OF TWO-LEVEL INHOMOGENEOUS QUANTUM ENSEMBLES}

In this section, we apply SLC to two-level inhomogeneous ensembles. Several groups of numerical experiments are given to evaluate the performance of SLC.

\section{A. Two-level inhomogeneous ensemble}

Consider a quantum ensemble consisting of two-level quantum systems (e.g., spins). The Pauli matrices $\sigma=\left(\sigma_{x}, \sigma_{y}, \sigma_{z}\right)$ are denoted as follows:

$\sigma_{x}=\left(\begin{array}{ll}0 & 1 \\ 1 & 0\end{array}\right), \quad \sigma_{y}=\left(\begin{array}{cc}0 & -i \\ i & 0\end{array}\right), \quad \sigma_{z}=\left(\begin{array}{cc}1 & 0 \\ 0 & -1\end{array}\right)$.

We let the free Hamiltonian be $H_{0}=\frac{1}{2} \sigma_{z}$ and its two eigenstates are denoted as $|0\rangle$ and $|1\rangle$. The control Hamiltonian is $H_{u}=\frac{1}{2} u_{1}(t) \sigma_{x}+\frac{1}{2} u_{2}(t) \sigma_{y}$. Then we have

$$
|\dot{\psi}(t)\rangle=-i H(t)|\psi(t)\rangle,
$$

where $\quad H(t)=H_{0}+H_{u}(t)=\frac{1}{2} \sigma_{z}+\frac{1}{2} u_{1}(t) \sigma_{x}+\frac{1}{2} u_{2}(t) \sigma_{y}$. For the inhomogeneous ensemble, the Hamiltonian of each member is described as

$$
H_{\omega, \theta}(t)=\omega H_{0}+\theta H_{u}(t) .
$$

The state of the quantum system can be represented as $|\psi(t)\rangle=$ $c_{0}(t)|0\rangle+c_{1}(t)|1\rangle$. Denote $C(t)=\left(c_{0}(t), c_{1}(t)\right)^{T}$, where $c_{0}(t)$ and $c_{1}(t)$ are complex amplitudes. We have

$$
i \dot{C}(t)=\left[H_{0}+H_{u}(t)\right] C(t) .
$$

To construct a generalized system for the training step, we select $N$ members $(n=1,2, \ldots, N)$ from the ensemble, and each satisfies

$$
\left(\begin{array}{c}
\dot{c}_{0, n}(t) \\
\dot{c}_{1, n}(t)
\end{array}\right)=\left(\begin{array}{cc}
0.5 \omega_{n} i & \theta_{n} f(u) \\
-\theta_{n} f^{*}(u) & -0.5 \omega_{n} i
\end{array}\right)\left(\begin{array}{l}
c_{0, n}(t) \\
c_{1, n}(t)
\end{array}\right),
$$

where $f(u)=u_{2}(t)-0.5 i u_{1}(t), \omega_{n} \in[1-\Omega, 1+\Omega]$, and $\theta_{n} \in[1-\Theta, 1+\Theta]$ have uniform distributions. The objective is to find a control $u(t)=\left\{u_{m}(t), m=1,2\right\}$ to drive all the inhomogeneous members from an initial state $\left|\psi_{0}\right\rangle=|0\rangle$, i.e., $C_{0}=(1,0)^{T}$, to the target state $\left|\psi_{\text {target }}\right\rangle=|1\rangle$, i.e., $C_{\text {target }}=$ $(0,1)^{T}$. We construct a generalized system for the training samples using Eq. (6) with the performance function $J_{N}(u)$ in Eq. (7).

The task is to find the control $u(t)$ to maximize the performance function $J_{N}(u)$. For a given small threshold $\epsilon>0$, if $J_{N}\left(u^{*}\right)>1-\epsilon$, then we have found a suitable candidate optimal control for the generalized system. We employ Algorithm 1 to find the optimal control $u^{*}(t)=$ $\left\{u_{m}^{*}(t), m=1,2\right\}$ for this generalized system. This optimal control is then applied to other randomly selected members to test its performance.

\section{B. Numerical results}

Several groups of numerical experiments are carried out on an inhomogeneous spin ensemble to demonstrate SLC. The parameter settings are as follows: $\Omega=0.2$ and $\Theta=0.2$; the target time is $T=2$, and the total time interval $[0, T]$ is divided equally into $Q=200$ time steps, $\Delta t=T / Q=0.01$; 

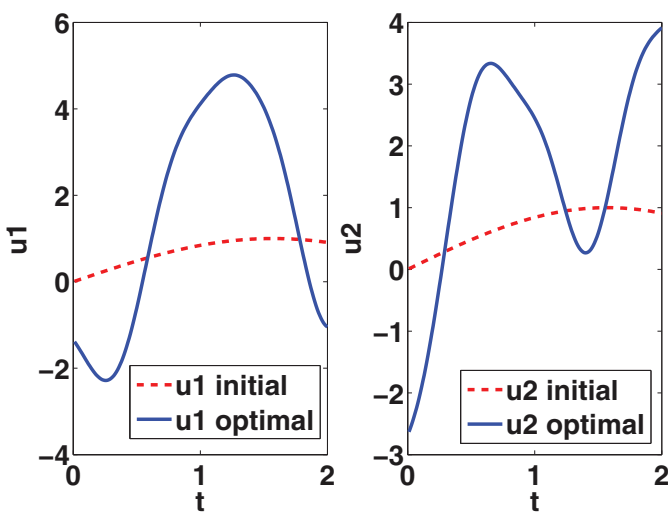

FIG. 2. (Color online) The learned optimal control strategy that maximizes $J_{N}(u)$ for the two-level ensemble with two controls.

the learning rate is set as $\eta^{k}=0.2$; the control strategy is initialized as $u^{k=0}(t)=\left\{u_{1}^{0}(t)=\sin t, u_{2}^{0}(t)=\sin t\right\}$.

\section{Performance with two controls}

To construct a generalized system for the inhomogeneous ensemble with parameter dispersion on both $\omega$ and $\theta$, we choose $N_{\Omega}=5$ and $N_{\Theta}=5$ such that $N=N_{\Omega} N_{\Theta}=25$ samples are employed in the learning phase. Using Eq. (8), we have

$$
\begin{aligned}
& \omega_{n}=1-0.2+\frac{0.2[2 \mathrm{fix}(n / 5)-1]}{5}, \\
& \theta_{n}=1-0.2+\frac{0.2[2(\bmod (n, 5)-1]}{5},
\end{aligned}
$$

where $\quad n=1,2, \ldots, 25, \quad$ fix $(x)=\max \{z \in \mathbb{Z} \mid z \leqslant x\}$, $\bmod (n, 5)=n-5 z \quad\left(z \in \mathbb{Z}\right.$ and $\left.\frac{n}{5}-1<z \leqslant \frac{n}{5}\right)$, and $\mathbb{Z}$ is the set of integers. We set $\epsilon=5 \times 10^{-5}$. The algorithm converges after around 380 iterations. The learned optimal control strategy is given as in Fig. 2, and the testing performance in Fig. 3 shows that the fidelities for the state transition lie in the interval of $[0.9985,1]$ with mean value of 0.9997. For comparison, if we use only one sample $(\omega=1, \theta=1)$ for training to obtain a control law, the testing performance gives fidelities that lie in $[0.9436,1]$ with a mean value of 0.9808 .

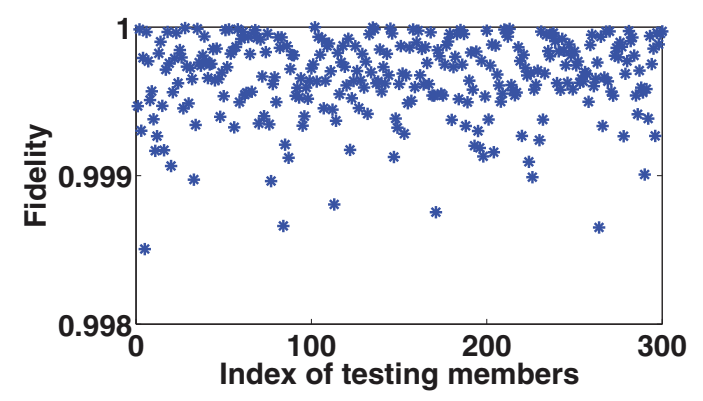

FIG. 3. (Color online) The testing performance (with respect to fidelity) of the learned optimal control strategy for the two-level ensemble with two controls (where $\omega$ and $\theta$ are randomly chosen with 300 pairs of values).
The numerical results show that SLC is effective for control design of the two-level inhomogeneous ensemble. The fidelities of the controlled state for the randomly selected members approach very near to 1 even with $\pm 20 \%$ parameter dispersion over a uniform distribution.

Using the optimal control strategy in Fig. 2, we randomly select several thousand members and present the state transition trajectories of the two-level ensemble on the Bloch sphere. For a two-level system on the Bloch sphere, its state can be represented using a vector $\mathbf{r}=(x, y, z)$ where $x=\operatorname{tr}\left\{|\psi\rangle\langle\psi| \sigma_{x}\right\}, \quad y=\operatorname{tr}\left\{|\psi\rangle\langle\psi| \sigma_{y}\right\}, \quad z=\operatorname{tr}\left\{|\psi\rangle\langle\psi| \sigma_{z}\right\}$. As shown in Fig. 4, although the trajectories of these randomly selected members considerably differ from each other due to the inhomogeneity of the ensemble, they are all successfully driven from the initial state $\left|\psi_{0}\right\rangle=|0\rangle$, i.e., $r_{0}=(0,0,1)$, to the same target state $\left|\psi_{\text {target }}\right\rangle=|1\rangle$, i.e., $r_{\text {target }}=(0,0,-1)$, with the high fidelity indicated above.

\section{Performance with $u_{1}(t)=u_{2}(t)$}

Here we consider the restriction of operating with only one control by setting $u(t)=u_{1}(t)=u_{2}(t)$ with the evolution equation of the ensemble being

$$
\left(\begin{array}{c}
\dot{c}_{0}(t) \\
\dot{c}_{1}(t)
\end{array}\right)=\left(\begin{array}{cc}
0.5 \omega i & \theta h(u) \\
-\theta h^{*}(u) & -0.5 \omega i
\end{array}\right)\left(\begin{array}{l}
c_{0}(t) \\
c_{1}(t)
\end{array}\right)
$$

where $h(u)=u(t)-0.5 i u(t)$. We apply the same SLC design method and parameter settings as in Sec. III.B.1 except that $\epsilon=2.0 \times 10^{-2}$. After around 500 iterations, the algorithm finds an optimal control field. The optimal control strategy is shown in Fig. 5, and Fig. 6 gives the testing performance of 300 randomly selected testing members, whose fidelities lie in $[0.9727,1]$ with a mean value of 0.9939 . Upon comparison with the case in Sec. III.B.1, the restricted control has very good but reduced fidelity.

\section{CONTROL OF THREE-LEVEL INHOMOGENEOUS QUANTUM ENSEMBLES}

In this section, we further demonstrate SLC with a $\Lambda$ type three-level inhomogeneous ensemble. We conclude this section with a summary of the state transition control fidelities for all the cases in the paper.

\section{A. Control of a $\Lambda$-type atomic ensemble}

We consider a $\Lambda$-type atomic ensemble and demonstrate the SLC design process. For a $\Lambda$-type atomic system [43,44], we assume that the initial state is $|\psi(t)\rangle=c_{1}(t)|1\rangle+c_{2}(t)|2\rangle+$ $c_{3}(t)|3\rangle$ and denote $C(t)=\left(c_{1}(t), c_{2}(t), c_{3}(t)\right)$ where $c_{i}(t)$ are complex amplitudes. We have

$$
i \dot{C}(t)=\left[H_{0}+H_{u}(t)\right] C(t) .
$$

We take $H_{0}=\operatorname{diag}(1.5,1,0)$ and choose $H_{1}$ and $H_{2}$ in the control Hamiltonian of Eq. (3) as follows [43]:

$$
H_{1}=\left(\begin{array}{lll}
0 & 0 & 0 \\
0 & 0 & 1 \\
0 & 1 & 0
\end{array}\right), \quad H_{2}=\left(\begin{array}{lll}
0 & 0 & 1 \\
0 & 0 & 0 \\
1 & 0 & 0
\end{array}\right)
$$



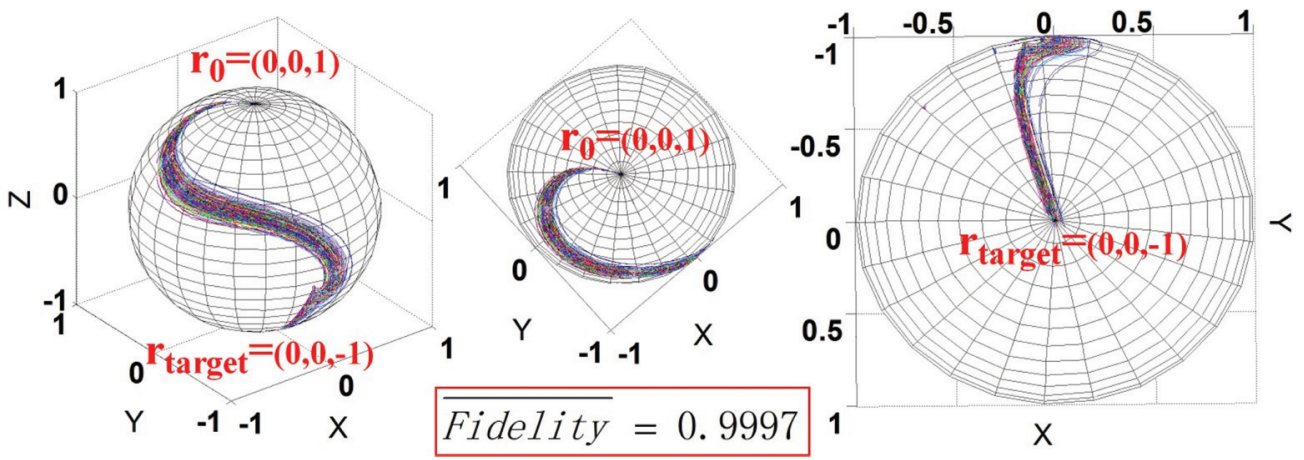

FIG. 4. (Color online) Demonstration of the state transition trajectories of the two-level ensemble with inhomogeneities due to both $\omega$ and $\theta$ using the learned optimal control strategy as shown in Fig. 2 for the state transition problem of guiding the initial state $r_{0}=(0,0,1)($ i.e., $\left.\left|\psi_{0}\right\rangle=|0\rangle\right)$ to the target state $r_{\text {target }}=(0,0,-1)$ (i.e., $\left|\psi_{\text {target }}\right\rangle=|1\rangle$ ).

To construct a generalized system for the SLC training step, we choose $N$ samples from the ensemble to form

$$
\begin{aligned}
\left(\begin{array}{l}
\dot{c}_{1, n}(t) \\
\dot{c}_{2, n}(t) \\
\dot{c}_{3, n}(t)
\end{array}\right)= & \left(\begin{array}{ccc}
-1.5 \omega_{n} i & 0 & -i \theta_{n} u_{2}(t) \\
0 & -\omega_{n} i & -i \theta_{n} u_{1}(t) \\
-i \theta_{n} u_{2}(t) & -i \theta_{n} u_{1}(t) & 0
\end{array}\right) \\
& \times\left(\begin{array}{c}
c_{1, n}(t) \\
c_{2, n}(t) \\
c_{3, n}(t)
\end{array}\right)
\end{aligned}
$$

where $\omega_{n} \in[1-\Omega, 1+\Omega]$ and $\theta_{n} \in[1-\Theta, 1+\Theta]$ have uniform distributions. The objective is to find a control strategy $u(t)=\left\{u_{m}(t), m=1,2\right\}$ to drive all the inhomogeneous members from $\left|\psi_{0}\right\rangle=\frac{1}{\sqrt{3}}(|1\rangle+|2\rangle+|3\rangle)$, i.e., $C_{0}=\left(\frac{1}{\sqrt{3}}, \frac{1}{\sqrt{3}}, \frac{1}{\sqrt{3}}\right)$, to $\left|\psi_{\text {target }}\right\rangle=|3\rangle$, i.e., $C_{\text {target }}=(0,0,1)$. We aim to maximize the performance function $J_{N}(u)$ in Eq. (7) and employ Algorithm 1 to find the optimal control $u^{*}(t)=\left\{u_{m}^{*}(t), m=\right.$ $1,2\}$ for this generalized system. Then the optimal control strategy is applied to other randomly selected members to test their performance.

\section{B. Numerical example}

We choose the parameter settings as follows: the control strategy is initialized with $u^{k=0}(t)=\left\{u_{m}^{0}(t)=\sin t, m=1,2\right\}$; $\epsilon=10^{-4}$; the other parameter settings are the same as those of

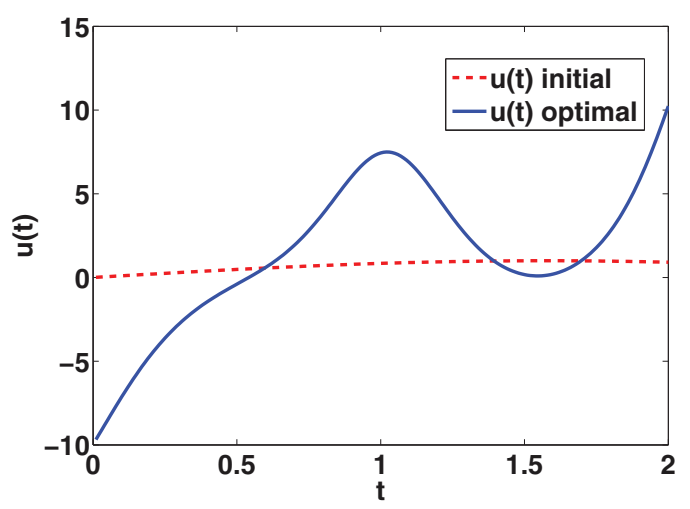

FIG. 5. (Color online) The learned optimal control that maximizes $J_{N}(u)$ for the two-level ensemble with $u_{1}(t)=u_{2}(t)=u(t)$. the numerical experiments for the spin ensemble in Sec. III. To construct a generalized system for the training step, we have the training samples selected as follows:

$$
\begin{aligned}
\omega_{n} & =1-0.2+\frac{0.2[2 \operatorname{fix}(n / 5)-1]}{5}, \\
\theta_{n} & =1-0.2+\frac{0.2[2 \bmod (n, 5)-1]}{5},
\end{aligned}
$$

where $n=1,2, \ldots, 25$. The algorithm converges after around 2000 iterations. The learned optimal control strategy is given in Fig. 7, and the testing results are given in Fig. 8, which shows that the fidelities for all the 300 testing members lie in the interval of $[0.9881,1]$ with the mean value of 0.9972 . For comparison, if we use only one sample $(\omega=1, \theta=1)$ for training to obtain a control, the testing performance gives fidelities that lie in $[0.8279,1]$ with the mean value of 0.9449 .

As a summary of the overall numerical tests of SLC, Fig. 9 shows the control performance (including some cases that have been explicitly shown above) for the aforementioned spin and $\Lambda$-type atomic ensembles. For the two-level inhomogeneous ensemble with parameter dispersion only in $\omega$, the fidelities of all the 300 testing members are excellent and lie in the interval of $\left[1-10^{-6}, 1\right]$. For the case with parameter dispersion only in $\theta$, the fidelities lie in the interval of $[0.9987,1]$ with the mean value 0.9994 . If only $u_{1}(t)=u_{2}(t)$ is allowed, the control performance is not as good as that with two controls $u_{1}(t)$ and $u_{2}(t)$. The collective numerical results further demonstrate the

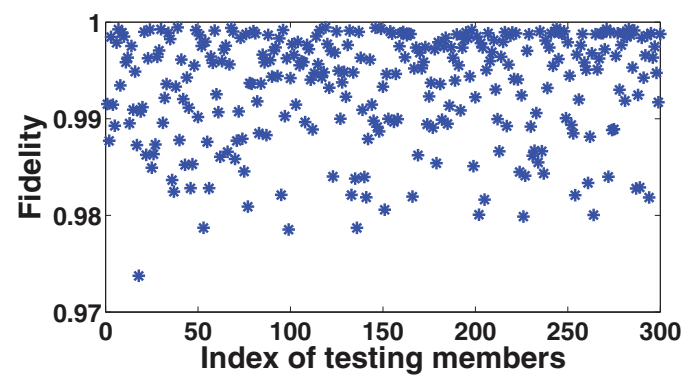

FIG. 6. (Color online) The testing performance (with respect to fidelity) of the learned optimal control for the two-level ensemble with $u_{1}(t)=u_{2}(t)=u(t)$ (where $\omega$ and $\theta$ are randomly chosen with 300 pairs of values). 

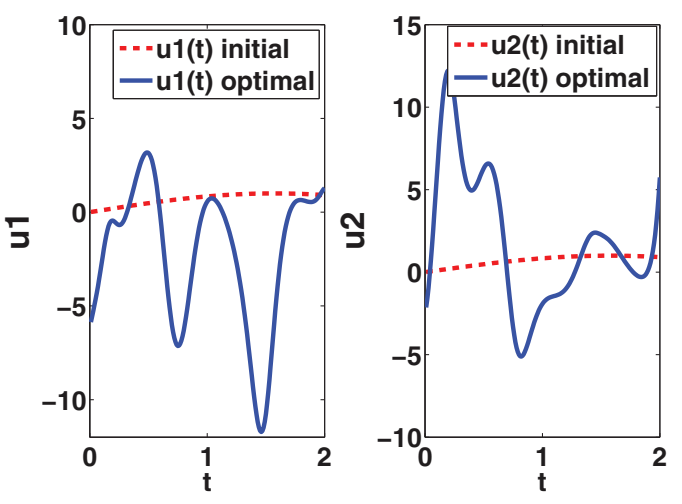

FIG. 7. (Color online) The learned optimal control strategy to maximize $J_{N}(u)$ for the $\Lambda$-type atomic ensemble.

theoretical predictions that ensemble control should be feasible [32-34].

\section{DISCUSSIONS AND CONCLUSIONS}

In this paper, we presented a systematic methodology for control design of inhomogeneous quantum ensembles. The proposed SLC method includes the steps of (i) training and (ii) testing. In the training step, the control is learned for a generalized system constructed from samples using a gradient flow based learning and optimization algorithm. In the process of testing, the control obtained in the first step is evaluated using additional randomly selected members. In this paper, we employed a general gradient flow based algorithm to find the control field. It is also straightforward to extend the proposed approach with the use of other specific gradient-type algorithms (e.g., GRAPE [29]). However, stochastic search algorithms such as genetic algorithms and differential evolution algorithms are not good candidates for this task under consideration since too many parameters need to be optimized and much more computation resources would be consumed using this class of algorithms. For the inhomogeneous parameters, we considered the case with a uniform distribution. Our method also works well for other distributions (e.g., Gaussian distribution). However, numerical results showed that even inhomogeneous parameters have a continuous Gaussian distribution; sampling members according to a relevant uniform distribution can achieve better

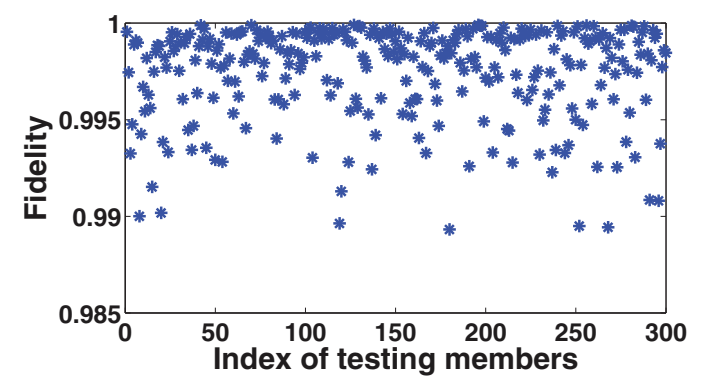

FIG. 8. (Color online) The testing performance (with respect to fidelity) of the learned optimal control strategy for the $\Lambda$-type atomic ensemble (where $\omega$ and $\theta$ are randomly chosen with 300 pairs of values).

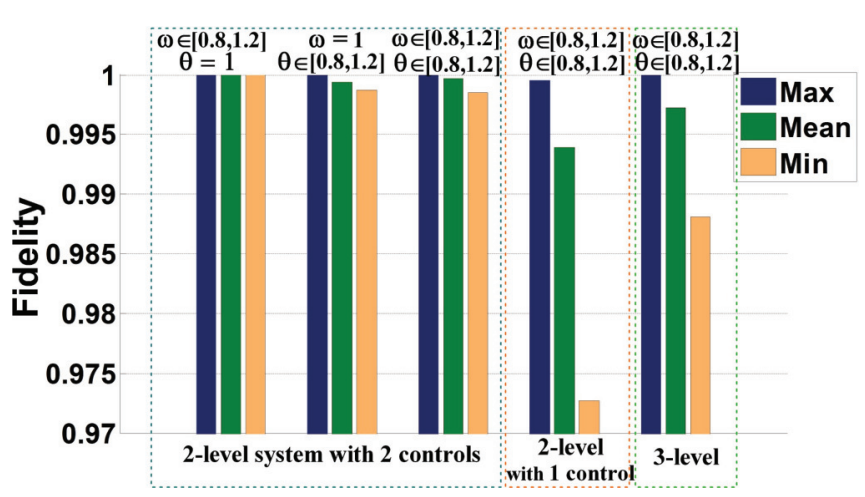

FIG. 9. (Color online) Control performance with respect to fidelity for the two-level and three-level inhomogeneous ensembles.

performance than sampling them according to this continuous Gaussian distribution. The reason could be that these members from a uniform distribution contain more information for learning the control field than those from the Gaussian distribution. Moreover, the proposed method can also be applied to multilevel systems with several inhomogeneous parameters. However, too many inhomogeneous parameters would make the algorithm difficult to converge. In the laboratory, one approach would be to first learn a control field offline using the SLC method and then generate and apply the control field to an inhomogeneous quantum ensemble.

\section{ACKNOWLEDGMENTS}

This work was supported by the Natural Science Foundation of China (Grants No. 61273327 and No. 61374092), by the Australian Research Council (DP130101658 and FL110100020), by the U.S. Department of Energy (Grant No. DE-FG02-02ER15344), by the NSF (Grant No. CHE0718610), and by the Army Research Office (Grant No. 0025020).

\section{APPENDIX: GRADIENT FLOW METHODS FOR QUANTUM ENSEMBLE CONTROL}

To get an optimal control strategy $u^{*}=\left\{u_{m}^{*}(t),(t \in\right.$ $[0, T]), m=1,2, \ldots, M\}$ for the generalized system Eq. (6), one technique is to follow the gradient of $J_{N}(u)$ in the steepest ascent direction.

In practical applications, we use the following discrete iteration rule to update the control field (for details relating

Algorithm 1 Gradient flow based iterative learning

1: Set the iteration index $k=0$

2: Choose a set of arbitrary controls $u^{k=0}(t)=\left\{u_{m}^{0}(t), m=1,2, \ldots, M\right\}, t \in[0, T]$

3: repeat steps 4-6 (corresponding to one iteration)

4: $\quad$ Compute the propagator $U_{\omega_{n}, \theta_{n}}(t)$ with $u^{k}(t)$ for all the training sample members $(n=1,2, \ldots, N)$

5: $\quad$ Update each control $u_{m}^{k+1}(t)=u_{m}^{k}(t)+\eta^{k} \delta_{m}^{k}(t)$ with $\delta_{m}^{k}(t)=\frac{2}{N} \sum_{n=1}^{N} \Im\left(\left\langle\psi_{\omega_{n}, \theta_{n}}(T) \mid \psi_{\text {target }}\right\rangle\left\langle\psi_{\text {target }}\left|A_{m}(t)\right| \psi_{0}\right\rangle\right)$ where $A_{m}(t)=U_{\omega_{n}, \theta_{n}}(T) U_{\omega_{n}, \theta_{n}}^{\dagger}(t) \theta_{n} H_{m} U_{\omega_{n}, \theta_{n}}(t)$ and $m=1,2, \ldots, M$ 6: $\quad k=k+1$

7: until the learning process ends (i.e., the algorithm converges)

8: The optimal control strategy $u^{*}=\left\{u_{m}^{*}\right\}=\left\{u_{m}^{k}\right\}, m=1,2, \ldots, M$ 
to gradient algorithms, see, e.g., $[5,28,39,41,42])$ :

$$
u^{k+1}(t)=u^{k}(t)+\eta^{k} \nabla J_{N}\left(u^{k}\right),
$$

where $\eta^{k}$ is the updating step size (learning rate) for the $k$ th iteration. By Eq. (7), we also obtain that

$$
\nabla J_{N}(u)=\frac{1}{N} \sum_{n=1}^{N} \nabla J_{\omega_{n}, \theta_{n}}(u) .
$$

In addition, we have

$\nabla J_{\omega_{n}, \theta_{n}}(u)=2 \Im\left(\left\langle\psi_{\omega_{n}, \theta_{n}}(T) \mid \psi_{\text {target }}\right\rangle\left\langle\psi_{\text {target }}\left|A_{1}(t)\right| \psi_{0}\right\rangle\right)$, where $A_{1}(t)=U_{\omega_{n}, \theta_{n}}(T) U_{\omega_{n}, \theta_{n}}^{\dagger}(t) \theta_{n} H_{1} U_{\omega_{n}, \theta_{n}}(t), \Im(\cdot)$ denotes the imaginary part of a complex number, and the propagator $U_{\omega_{n}, \theta_{n}}(t)$ satisfies

$$
\frac{d}{d t} U_{\omega_{n}, \theta_{n}}(t)=-i H_{\omega_{n}, \theta_{n}}(t) U_{\omega_{n}, \theta_{n}}(t), \quad U(0)=\mathrm{Id} .
$$

The gradient flow method can be generalized to the case with $M>1$ as shown in Algorithm 1 .
[1] D. Dong and I. R. Petersen, IET Control Theory Appl. 4, 2651 (2010).

[2] C. Altafini and F. Ticozzi, IEEE Trans. Automat. Control 57, 1898 (2012).

[3] H. M. Wiseman and G. J. Milburn, Quantum Measurement and Control (Cambridge University Press, Cambridge, 2010).

[4] B. Qi, Z. B. Hou, L. Li, D. Dong, G. Y. Xiang, and G. C. Guo, Sci. Rep. 3, 3496 (2013).

[5] C. Brif, R. Chakrabarti, and H. Rabitz, New J. Phys. 12, 075008 (2010).

[6] M. Mirrahimi, P. Rouchon, and G. Turinici, Automatica 41, 1987 (2005).

[7] X. Wang and S. G. Schirmer, IEEE Trans. Automat. Control 55, 2259 (2010).

[8] H. Rabitz, R. de Vivie-Riedle, M. Motzkus, and K. Kompa, Science 288, 824 (2000).

[9] N. Khaneja, R. Brockett, and S. J. Glaser, Phys. Rev. A 63, 032308 (2001).

[10] H. M. Wiseman and G. J. Milburn, Phys. Rev. Lett. 70, 548 (1993).

[11] R. van Handel, J. K. Stockton, and H. Mabuchi, IEEE Trans. Automat. Control 50, 768 (2005).

[12] B. Qi and L. Guo, Syst. Control Lett. 59, 333 (2010).

[13] J. Zhang, L. Greenman, X. Deng, and K. B. Whaley, arXiv:1210.7972 [quant-ph].

[14] C. Sayrin, I. Dotsenko, X. Zhou, B. Peaudecerf, T. Rybarczyk, S. Gleyzes, P. Rouchon, M. Mirrahimi, H. Amini, M. Brune, J.-M. Raimond, and S. Haroche, Nature (London) 477, 73 (2011).

[15] M. R. James, H. I. Nurdin, and I. R. Petersen, IEEE Trans. Automat. Control 53, 1787 (2008).

[16] D. Dong and I. R. Petersen, New J. Phys. 11, 105033 (2009).

[17] D. Dong and I. R. Petersen, Automatica 48, 725 (2012).

[18] D. Dong, C. Zhang, H. Rabitz, A. Pechen, and T. J. Tarn, J. Chem. Phys. 129, 154103 (2008).

[19] R. S. Judson and H. Rabitz, Phys. Rev. Lett. 68, 1500 (1992).

[20] D. G. Cory, A. F. Fahmy, and T. F. Havel, Proc. Natl. Acad. Sci. USA 94, 1634 (1997).

[21] L. M. Duan, M. D. Lukin, J. I. Cirac, and P. Zoller, Nature (London) 414, 413 (2001).
[22] G. Bensky, D. Petrosyan, J. Majer, J. Schmiedmayer, and G. Kurizki, Phys. Rev. A 86, 012310 (2012).

[23] J. S. Li, J. Ruths, T. Y. Yu, H. Arthanari, and G. Wagner, Proc. Natl. Acad. Sci. USA 108, 1879 (2011).

[24] S. J. Glaser, T. Schulte-Herbrüggen, M. Sieveking, O. Schedletzky, N. C. Nielsen, O. W. Sørensen, and C. Griesinger, Science 280, 421 (1998).

[25] C. Altafini, IEEE Trans. Automat. Control 52, 2019 (2007).

[26] M. H. Levitt, Prog. Nucl. Magn. Reson. Spectrosc. 18, 61 (1986).

[27] J. S. Li and N. Khaneja, Phys. Rev. A 73, 030302(R) (2006).

[28] T. E. Skinner, T. O. Reiss, B. Luy, N. Khaneja, and S. J. Glaser, J. Magn. Reson. 163, 8 (2003).

[29] N. Khaneja, T. Reiss, and C. Kehlet, T. Schulte-Herbrüggen, and S. J. Glaser, J. Magn. Reson. 172, 296 (2005).

[30] L. M. K. Vandersypen and I. L. Chuang, Rev. Mod. Phys. 76, 1037 (2004).

[31] G. Turinici and H. Rabitz, Phys. Rev. A 70, 063412 (2004).

[32] G. Turinici, V. Ramakhrishna, B. Li, and H. Rabitz, J. Phys. A 37, 273 (2004).

[33] H. Rabitz and G. Turinici, Phys. Rev. A 75, 043409 (2007).

[34] J. S. Li and N. Khaneja, IEEE Trans. Automat. Control 54, 528 (2009).

[35] J. Ruths and J. S. Li, J. Chem. Phys. 134, 044128 (2011).

[36] J. Ruths and J. S. Li, IEEE Trans. Automat. Control 57, 2021 (2012).

[37] P. Owrutsky and N. Khaneja, Phys. Rev. A 86, 022315 (2012).

[38] K. Beauchard, P. S. P. da Silva, and P. Rouchon, Automatica 48, 68 (2012)

[39] R. Long, G. Riviello, and H. Rabitz, IEEE Trans. Automat. Control 58, 2665 (2013).

[40] M. A. Nielsen and I. L. Chuang, Quantum Computation and Quantum Information (Cambridge University Press, Cambridge, 2000).

[41] H. Jirari and W. Pötz, Phys. Rev. A 72, 013409 (2005).

[42] J. Roslund and H. Rabitz, Phys. Rev. A 79, 053417 (2009).

[43] S. C. Hou, M. A. Khan, X. X. Yi, D. Dong, and I. R. Petersen, Phys. Rev. A 86, 022321 (2012).

[44] J. Q. You and F. Nori, Nature (London) 474, 589 (2011). 